\title{
Research on the Offensive and Defensive Strength and Competition Pattern of Each Team in the CBA Season 2015-2016 League

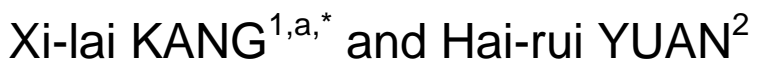 \\ ${ }^{1,2}$ School of Physical Education, Shaanxi Normal University, Shaanxi, Xi'an, 710119 \\ akangx185@snnu.edu.cn
}

Keywords: CBA, Offense-defense comparison, Competition pattern, Comparative analysis.

\begin{abstract}
The authors of this essay adopt methods such as documentary data, video analysis, mathematical statistics, RSR comprehensive evaluation to comprehensively analyze the offensive and defensive strengths of over 20 teams in the CBA season, 2015-2016 League. Studies reveal that at the offensive end the top six teams from League Ranking remain the first six in terms of their RSR value. Among the 20 competing teams, the Xin-jiang Team is the only one who's offensive RSR reaches Level A. While for these seven teams, namely, teams of Liao-ning, Si-chuan, Guang-dong, Shan-dong, Guang-sha, Zhe-jiang and Fu-jian, their offensive RSR is Level B. As for Ba-yi Team, Level E. At defensive end, of the top eight teams, in terms of their League scores, seven teams' RSR can be classified as Level B. At the offense and defense end, teams of Liao-ning and Shan-dong are the two whose performance is quite outstanding. The correlation coefficient for teams' score ranking and offense-defense value is respectively $r=-0.902(p<0.01)$.
\end{abstract}

Basketball in a combat sport in which attack and defense, as two basic forms, take place in turn. The characteristic of attacking and defending enables basketball to have attacking and defending to take place in turn, but at the same time, attacking and defending are relatively independent, and both enjoy equally important strategic positions. The basic requirement for basketball competition is to be capable of attacking and defending, a balance of attacking and defending. This can also reflect whether a team is mature and powerful.[1] The CBA Season 2015-2016 League ended on March 20th, 2016 with the Si-chuan Team as the final champion. The object of study is technical index of regular season of the 20 competing teams in the CBA Season 2015-2016 League. This study attempts to discuss the differences of the competing teams in terms of their attacking and defending skills, in the hope that this study will be beneficial for future training and matches so that the overall competition level and influence of CBA League will be further improved.

\section{Object of Study}

Technical index of attacking and defending of the 20 competing teams in the CBA Season 2015-2016 League.

\section{Method}

This study will adopt such methods as documentary data, video analysis, mathematical statistics and RSR comprehensive evaluation.

Rank and analogy method is also referred to as RSR, which is an evaluation method 
using the mean value of lines or columns as the criterion to reflect a comprehensive evaluation with various measurement units and indicators. Its calculation formula is: $\mathrm{RSR}=\sum R /(\mathrm{m} \times \mathrm{n})$, in which" $\sum R$ " means the rank and value of the evaluated, " $\mathrm{m}$ " is the number of evaluation index, " $n$ " refers to the competing teams. With a bigger value of RSR, its comprehensive strength is better. Research has shown that a bigger RSR value means higher comprehensive level[1]. This study, adopting five evaluation indexes as well as establishing evaluation standards of team strength, uses RSR to respectively analyze offense-defense strength of the 20 competing teams in the CBA Season 2015-2016 League. It is shown in Table 1.

Table 1. RSR evaluation scale standards

\begin{tabular}{ccccc}
\hline A & B & C & D & E \\
\hline More than 0.80 & $0.79 \sim 0.60$ & $0.59 \sim 0.40$ & $0.39 \sim 0.20$ & Below 0.19 \\
\hline
\end{tabular}

\section{Result and Analysis}

\section{RSR Analysis of Offensive and Defensive Strength of Each Competing Team in the CBA Season 2015-2016 League}

In order to have a detailed meticulous study of attacking and defending strength of each competing team in the CBA Season 2015-2016 League, the authors of this paper will choose such eight attacking indexes as scoring, overall shooting rate, two-point goal rate, three-point goal rate, penalty shots, offensive rebounds, assists, errors, in which errors are considered as a low optimal index.[2] The chosen five defending indexes include defensive rebounds, blocks, points losing, ball stealing, fouls, in which points losing and fouls belong to low optimal indexes.[3] In order for the study to have consistency in rank orientation, the authors of this study adopt reversed assignment for those low optimal indexes.

Table 2. Competition Record of the CBA Season 2015-2016 League

\begin{tabular}{|c|c|c|c|c|c|c|c|c|}
\hline Ranking & Name of Team & Matches & Winning & Losing & $\begin{array}{l}\text { Scores } \\
\text { Gained }\end{array}$ & Scores Lost & $\begin{array}{c}\text { Net Gained } \\
\text { Scores }\end{array}$ & $\begin{array}{l}\text { Rate of Scores } \\
\text { Gaining-Losing }\end{array}$ \\
\hline 1 & Liao-ning & 38 & 31 & 7 & 4226 & 3789 & 437 & 1.12 \\
\hline 2 & Xin-jiang & 38 & 30 & 8 & 4272 & 3845 & 427 & 1.11 \\
\hline 3 & Si-chuan & 38 & 30 & 8 & 4360 & 4034 & 326 & 1.08 \\
\hline 4 & Guang-dong & 38 & 28 & 10 & 4168 & 3735 & 433 & 1.12 \\
\hline 5 & Shan-dong & 38 & 28 & 10 & 4238 & 3913 & 325 & 1.08 \\
\hline 6 & Guang-sha & 38 & 27 & 11 & 4357 & 4081 & 276 & 1.07 \\
\hline 7 & Beijing & 38 & 23 & 15 & 3928 & 3747 & 181 & 1.05 \\
\hline 8 & Zhe-jiang & 38 & 22 & 16 & 4099 & 4123 & -24 & 1.00 \\
\hline 9 & Shen-zhen & 38 & 19 & 19 & 3851 & 3969 & -118 & 0.98 \\
\hline 10 & Shan-xi & 38 & 18 & 20 & 4050 & 4109 & -59 & 0.99 \\
\hline 11 & Jiang-su & 38 & 18 & 20 & 3927 & 3961 & -34 & 1.00 \\
\hline 12 & Shanghai & 38 & 18 & 20 & 3720 & 3786 & -66 & 0.99 \\
\hline 13 & Fu-jian & 38 & 16 & 22 & 4074 & 4128 & -54 & 0.99 \\
\hline 14 & Qing-dao & 38 & 16 & 22 & 4334 & 4464 & -130 & 0.97 \\
\hline 15 & Bei-kong & 38 & 13 & 25 & 3944 & 4063 & -119 & 0.97 \\
\hline 16 & Tong-xi & 38 & 12 & 26 & 3889 & 4179 & -290 & 0.93 \\
\hline 17 & $\mathrm{Ji}-\mathrm{lin}$ & 38 & 10 & 28 & 3903 & 4252 & -349 & 0.92 \\
\hline 18 & Tian-jin & 38 & 8 & 30 & 4029 & 4411 & -382 & 0.91 \\
\hline 19 & Ba-yi & 38 & 7 & 31 & 3297 & 3694 & -397 & 0.90 \\
\hline 20 & Fo-shan & 38 & 6 & 32 & 4203 & 4586 & -383 & 0.92 \\
\hline
\end{tabular}




\section{RSR Analysis of Attacking Technology of Each Competing Team of the CBA Season 2015-2016 League}

From Table 2 and Table 3, it is easy to find out that among all the competing teams of the CBA Season 2015-2016 League, Xin-jiang Team is the only one who's attacking capability somehow has reached Level A, with a RSR value of 0.80 . Nevertheless, its advantage is not outstanding compared with Liao-ning Team (0.74), Si-chuan Team (0.78), Guang-dong Team (0.71), Shan-dong Team (0.78) or Guang-sha (0.73). All these six teams have presented their unique characteristics in offensive technique. Within this season, Xin-jiang Team, after joining of the player called Li Gen, preformed excellently in two-point shots as well as in overall shots but did not do a good job in challenging offensive rebounds. However, Liao-ning Team showed great teamwork spirit in striving for offensive rebounds and assists. Si-chuan Team, with three foreign players' joining the team, revealed powerful capability in scoring. Guang-dong Team did a great job in two-point shots, three-point shots and assists, but on the other hand, because its players of the core team get much older and the foreign player didn't perform as expected, they lagged far behind teams of the same level in offensive rebounds and error. Both Shan-dong Team with the introduction of the super foreigner player Beasley, and Guang-sha Team with a great number of young and talented players performed very well in various attacking fields. The attacking level of Fu-jian Team, Qing-dao Team and Zhe-jiang all reached Level B. The attacking level of Beijing Team, Shen-zhen Team, Shan-xi Team, Jiang-su Team, Bei-kong Team and Fo-shan was Level C, with RSR value respectively $0.58,0.40,0.48,0.47,0.46,0.5$, which indicates that their attacking ability was at about middle and lower levels. Surprisingly, Beijing Team, the grand champion of last season, ranked only 11th this time with a very low attacking RSR value of 0.58 . Except for its good performance in three-point shots (ranked 2nd) and error control (ranked 3rd), Beijing Team played quite badly this season. From video observation, this was closely to do with Marbury's aging, Sun Yue's injuries and Zhu Yanxi's absence from the whole season. Of all the 20 competing teams, four teams' attacking was Level B, namely teams of Shanghai (0.31), Tong-xi (0.28), Ji-lin (0.32), Tian-jin(0.27). As a matter of fact, their ranking and performance are quite consistent. Among all the competing teams, Ba-yi Team, in terms of attacking level, was ranked the last and is the only team classified as Class E, with an exceptionally low RSR value of 0.18. Actually, of all the eight attacking indicators, six of them from Ba-yi Team are the lowest or the last, a seventh indicator is the last but one. From video observation, it is easy to find out that Ba-yi Team doesn't have any foreign player on the team, nor does it have any outstanding domestic player. As a consequence, at the offensive end, this team is lacking in opportunity makers, which will surely lead to more defensive pressure when an attack comes to an end and will thus further result in low efficiency of scoring. So, Ba-yi's average scores per match were only 86.76 , far behind other competing teams. While in the case of Fo-shan Team, its RSR ranking was No. 10 out of 20 competing teams; however, its achievement was the last one owing to its lousy defense. As for the correlation coefficient of RSR value ranking between ranking and offensive indicators of all the competing teams in the CBA Season 2015-2016 League, $\mathrm{r}=-0.807(\mathrm{p}<0.01)$, the two are significantly correlated (Table 4$)$, which indicates that the RSR values of offensive indicators of all the competing teams are able to objectively reflect the teams' offensive strength. 
Table 3. Analysis of Attacking Indexes of Each Competing Team of the CBA Season 2015-2016 League

\begin{tabular}{|c|c|c|c|c|c|c|c|c|c|c|c|c|c|}
\hline Ranking & Team & Scores & Rank & $\begin{array}{l}\text { Shot } \\
\text { rate } \%\end{array}$ & Rank & $\begin{array}{l}\text { Two-point } \\
\text { shots } \%\end{array}$ & Rank & $\begin{array}{l}\text { Three-point } \\
\text { shot } \%\end{array}$ & Rank & $\begin{array}{l}\text { Penalty } \\
\text { shots }\end{array}$ & Rank & Assists & Rank \\
\hline 1 & Liao-ning & 111.21 & 15 & 48.6 & 13 & 53.77 & 11 & 38.52 & 17 & 15.13 & 5.5 & 20.2 & 20 \\
\hline 2 & Xin-jiang & 112.42 & 17 & 53.09 & 20 & 59.08 & 20 & 38.35 & 16 & 18.21 & 16 & 17.1 & 14 \\
\hline 3 & Si-chuan & 114.74 & 20 & 48.98 & 14 & 56.53 & 17 & 36.11 & 9 & 18.68 & 17 & 17.9 & 15.5 \\
\hline 4 & Guang-dong & 109.68 & 13 & 50.79 & 19 & 56.58 & 18 & 39.94 & 20 & 14.97 & 4 & 19.6 & 18.5 \\
\hline 5 & Shan-dong & 111.58 & 16 & 50.44 & 18 & 56.2 & 16 & 38.33 & 15 & 17.18 & 12 & 18.2 & 17 \\
\hline 6 & Guang-sha & 114.66 & 19 & 49.95 & 16 & 56.67 & 19 & 35.94 & 8 & 17.34 & 13 & 19.6 & 18.5 \\
\hline 7 & Beijing & 103.37 & 7 & 46.66 & 7 & 51.05 & 6 & 39.08 & 19 & 18.03 & 15 & 16.9 & 13 \\
\hline 8 & Zhe-jiang & 107.89 & 12 & 49.19 & 15 & 54.86 & 15 & 34.89 & 4 & 19.79 & 18 & 15.9 & 8 \\
\hline 9 & Shen-zhen & 101.34 & 3 & 46.31 & 5 & 52.72 & 8 & 36.57 & 12 & 16.42 & 8 & 16.0 & 9.5 \\
\hline 10 & Shan-xi & 106.58 & 10 & 47.06 & 8 & 53.36 & 9.5 & 36.37 & 10 & 12.68 & 1 & 17.9 & 15.5 \\
\hline 11 & Jiang-su & 103.34 & 6 & 47.46 & 10 & 53.36 & 9.5 & 36.81 & 13 & 17.61 & 14 & 15.8 & 7 \\
\hline 12 & Shang-hai & 97.89 & 2 & 44.4 & 3 & 49.92 & 2 & 34.42 & 3 & 16.45 & 9 & 13.3 & 3 \\
\hline 13 & Fu-jian & 107.21 & 11 & 49.97 & 17 & 54.6 & 14 & 39.02 & 18 & 16.55 & 10 & 15.2 & 5 \\
\hline 14 & Qing-dao & 114.05 & 18 & 46.37 & 6 & 50.19 & 3 & 37.21 & 14 & 23.13 & 20 & 16.3 & 12 \\
\hline 15 & Bei-kong & 103.79 & 8 & 48.39 & 12 & 53.96 & 12 & 35.71 & 6 & 16.71 & 11 & 16.2 & 11 \\
\hline 16 & Tong-xi & 102.42 & 4 & 45.91 & 4 & 50.39 & 5 & 36.51 & 12 & 15.82 & 7 & 15.3 & 6 \\
\hline 17 & Ji-lin & 102.71 & 5 & 47.71 & 11 & 54.29 & 13 & 33.26 & 2 & 12.92 & 2 & 16.0 & 9.5 \\
\hline 18 & Tian-jin & 106.03 & 9 & 44.03 & 2 & 50.25 & 4 & 35.1 & 5 & 14.66 & 3 & 12.4 & 1 \\
\hline 19 & Ba-yi & 86.76 & 1 & 42.09 & 1 & 49.92 & 1 & 29.24 & 1 & 15.13 & 5.5 & 12.9 & 2 \\
\hline 20 & Fo-shan & 110.61 & 14 & 47.41 & 9 & 52.25 & 7 & 35.85 & 7 & 21.87 & 19 & 14.9 & 4 \\
\hline
\end{tabular}

Table 3 (Continued)

\begin{tabular}{ccccccccc}
\hline Ranking & Team & Offensive Rebounds & Rank & Errors & Rank & RSR & Level & Sorting \\
\hline 1 & Liao-ning & 16.58 & 20 & 12.5 & 17 & 0.74 & B & 4 \\
2 & Xin-Jiang & 11.21 & 9 & 12.9 & 15.5 & 0.80 & A & 1 \\
3 & Si-chuan & 14.16 & 18 & 13.1 & 14 & 0.78 & B & 2 \\
4 & Guang-dong & 10.89 & 6 & 14.9 & 5 & 0.71 & B & 6 \\
5 & Shan-dong & 11.92 & 12 & 12.8 & 19 & 0.78 & B & 2 \\
6 & Guang-sha & 13.74 & 15 & 14.3 & 8.5 & 0.73 & B & 5 \\
7 & Beijing & 11.05 & 7 & 12.3 & 18.5 & 0.58 & C & 11 \\
8 & Zhe-jiang & 12.74 & 14 & 14.2 & 10 & 0.60 & B & 8 \\
9 & Shen-zhen & 10.61 & 4 & 12.9 & 15.5 & 0.41 & C & 14 \\
10 & Shan-xi & 10.13 & 2 & 14.3 & 8.5 & 0.40 & C & 15 \\
11 & Jiang-su & 11.55 & 10 & 14.7 & 6 & 0.47 & C & 12 \\
12 & Shanghai & 13.89 & 16 & 14 & 11 & 0.31 & D & 17 \\
13 & Fu-jian & 11.13 & 8 & 13.5 & 13 & 0.60 & B & 8 \\
14 & Qing=dao & 14.47 & 19 & 13.9 & 12 & 0.65 & B & 7 \\
15 & Bei-kong & 11.68 & 11 & 15.7 & 2 & 0.46 & C & 13 \\
16 & Tong-xi & 10.34 & 3 & 15.6 & 3 & 0.28 & D & 18 \\
17 & Ji-lin & 10.79 & 5 & 15 & 4 & 0.32 & D & 16 \\
18 & Tian-jin & 9.42 & 1 & 12.3 & 18.5 & 0.27 & D & 19 \\
19 & Ba-yi & 14.03 & 17 & 16.4 & 1 & 0.18 & E & 20 \\
20 & Fo-shan & 12.66 & 13 & 14.6 & 7 & 0.5 & C & 10 \\
\hline
\end{tabular}

Note: When the data get bigger, rank assignment gets bigger too; reversed assignment is used in error statistics.

\section{RSR Analysis of Defensive Technology of Competing Teams in the CBA Season 2015-2016 League}

A popular saying goes in the basketball circle in the USA, "Putting on a show depends on attacking, while winning a game depends on defending.[4] So, we can clearly see the importance of defending in modern basketball matches. From Table 1 and Table 4, the attacking capability of the 20 competing teams in the CBA Season 2015-2016 League can be divided into four levels, namely, Level B, Level C, Level D and Level E. Unfortunately, none of the teams can reach Level A in terms of its defending capability, which indicates that there is no such team with defending dominance. In the playoffs, seven teams out of eight reached Level B for their defending capability, which further convinces us of the saying "Winning a game depends on defending." In the season, the best defenders were Guang-dong Team and Liao-ning Team with RSR value of 0.75 and 0.73 respectively. Of all the Level B teams, Xin-jiang Team's 
defending RSR value was as low as 0.57, even lower than Ba-yi's 0.66. From video observation, we can see that it was closely to do with their starting defender, Liu Wei's aging, as well as their foreign player's bad defending habits. The whole team was overly dependent on Zhou Qi's inside blocking, while there was not a nice defensive system within the team, which was surely hidden dangers for Xin-jiang Team in the playoffs. Beijing Team, though with a pretty low attacking RSR value, was able to participate in the play-off mainly because of its overall defensive strength which was ranked as high as No. 3, with a defending RSR value of 0.68 . Here, it needs to be mentioned that Ba-yi Team's defending capability ranked No. 6, with a very low average loss of 97.21 points per match which ranked first among all the competing teams. From video observation, we can see that Ba-yi Team, owing to losing foreign players' powerful support in attacking, depended on defending. All the players are of very active defensive attitude and well-trained defensive habits; furthermore, the whole team possesses a very good defensive system. In this sense, Ba-yi Team has set a very good example for other teams. Of the twenty competing teams, six teams' defensive capability is classified as Level $\mathrm{C}$ with, respectively, defending RSR value of 0.42 (Zhe-jiang Team), 0.53 (Shen-zhen), 0.48 (Shan-xi), 0.53 (Jiang-su), 0.42 (Bei-kong) and 0.46 (Tong-xi). Defending level of teams of Qing-dao, Ji-lin and Tian-jin belong to Level D, while Fo-shan Team's defending RSR value was as low as 0.18 and that's the major reason why this team ranked last. Of all the competing teams in the CBA Season 2015-2016 League, their average defending RSR value was 0.52 , an equivalent of Level C, which means that the whole league's defending capability was rather low. In the CBA Season 2015-2016 League, the coefficient of association of each competing team's ranking and its defending RSR value sequencing is $r=-0.704(\mathrm{p}<0.01)$, which means that the two are of significant correlation (Table 6) and each team's defensive indicators can objectively reflect its defensive strength.

\section{RSR Analysis of Each Competing Team's Attacking and Defending Technology in the CBA Season 2015-2016 League}

Basketball is a sport combining offensive and defensive technology, so a single analysis of offensive indicators or defensive indicators can only analyze certain characteristics of a basketball team.[5] An objective evaluation of a team's offensive and defensive strength requires RSR analysis of both offensive and defensive technology. From Table 5, all the competing teams' comprehensive offensive and defensive strength in the CBA Season 2015-2016 League can be classified into five levels. The offensive and defensive strength of the top five teams in the regular season has reached Level A. Teams of Liao-ning, Guang-dong and Shan-dong have demonstrated balanced strength in both attack and defense, while teams of Si-chuan and Xin-jiang have shown upper-middle level in defense and superb capability in attack. And as for another two teams in the playoffs, namely, teams of Beijing and Zhe-jiang, their offensive and defensive RSR value were respectively 0.69 and 0.45 ,still quite a disparity compared with those teams of Level A. And that's why these two teams were eliminated in the first round of the playoffs. Of the twenty competing teams, seven teams' offensive and defensive level was of Level C, the middle level, while these six teams, namely, teams of Shan-xi, Bei-kong, Tong-xi, Ji-lin, Ba-yi and Fo-shan, were of Level D, with RSR values ranging from 0.38-0.28. Compared with the top teams, these six teams lag far behind either in attacks or in defense. Both offensive RSR value and defensive RSR value of Tian-jin Team ranked the last but one and its comprehensive strength is the worst of all the competing teams. 
Table 5 reveals that for most teams whose comprehensive strength are of Level A or B, when the offensive level is almost the same, they enjoy a bigger defensive RSR value; and if the offensive and defensive RSR value is bigger, its ranking will be at the very top of the list. For instance, Xin-jiang Team's offensive RSR value is 0.80 , ranked No. 1 among all the competing teams, but its defensive RSR value is 0.57 , ranked only No. 9. Although its offensive and defensive RSR value has reached 0.80 , and was ranked No. 2 in the regular season, it was defeated by Si-chuan Team (whose defensive RSR value is 0.61 with a ranking of No. 8) in the semi-final of the playoffs. Liao-ning Team and Guang-dong Team are the top 2 teams in defense, so they defeated all their rivals in the playoffs and their semi-final was inextricably involved. Beijing Team's offensive strength was ranked only No. 11; nevertheless, its excellent defense not only enabled it to enter the playoffs, but also created a lot of stress to Xin-jiang Team which ranked No. 2. For those teams from Level C and D, both of their offensive level and their defensive level are rather low, so their ranking and performance in attacks or defense are quite consistent. The reason why Ba-yi Team and Fo-shan Team were ranked the last two in the League is because both of them were suffering from their fatal defects. Compared with the other 19 competing teams in the League, Ba-yi teams lagged far behind in terms of offensive strength while Fo-shan Team also lagged far behind in terms of defensive strength. In the CBA Season 2015-2016 League, the coefficient of association of each competing team's ranking and its defending RSR value sequencing is $\mathrm{r}=-0.902(\mathrm{p}<0.01)$, which means that the two are of significant correlation (Table 6) and each team's offensive and defensive RSR values are able to objectively reflect its offensive and defensive strength.

Table 4. Statistical Analysis of Defending Indicators of All the Competing Teams in the CBA Season

2015-2016 League

\begin{tabular}{|c|c|c|c|c|c|c|c|c|c|c|c|c|c|c|}
\hline Ranking & Team & $\begin{array}{l}\text { Defensive } \\
\text { Blocking }\end{array}$ & Rank & Blocks & Rank & $\begin{array}{c}\text { Scores } \\
\text { lost }\end{array}$ & Rank & Fouls & Rank & Steals & Rank & RSR & Ranking & Class \\
\hline 1 & Liao-ning & 29.41 & 17 & 3.18 & 12 & 99.71 & 16 & 23.22 & 10 & 9.56 & 18 & 0.73 & 2 & B \\
\hline 2 & Xin-jiang & 25.61 & 7 & 4.97 & 20 & 101.18 & 15 & 21.21 & 14 & 7.95 & 11 & 0.57 & 9 & B \\
\hline 3 & Si-chuan & 31.59 & 19 & 2.86 & 10 & 106.16 & 11 & 21.86 & 13 & 7.63 & 8 & 0.61 & 8 & B \\
\hline 4 & Guang-dong & 26.1 & 10 & 3.06 & 12 & 98.29 & 19 & 20.19 & 18 & 9.1 & 16 & 0.75 & 1 & B \\
\hline 5 & Shan-dong & 29.47 & 18 & 2.87 & 11 & 102.98 & 14 & 24.41 & 6 & 10.2 & 19 & 0.68 & 3 & B \\
\hline 6 & Guang-sha & 37.72 & 20 & 4.23 & 18 & 107.40 & 9 & 21.01 & 15 & 7.23 & 4 & 0.66 & 6 & B \\
\hline 7 & Beijing & 28.54 & 15 & 3.61 & 14 & 98.61 & 18 & 24.31 & 7 & 8.85 & 14 & 0.68 & 3 & B \\
\hline 8 & Zhe-jiang & 26.92 & 12 & 2.62 & 8 & 108.50 & 7 & 25.35 & 3 & 8.27 & 12 & 0.42 & 15 & $\mathrm{C}$ \\
\hline 9 & Shen-zhen & 27.39 & 14 & 4.18 & 17 & 104.45 & 12 & 24.08 & 9 & 7.11 & 1 & 0.53 & 10 & $\mathrm{C}$ \\
\hline 10 & Shan-xi & 23.24 & 2 & 3.68 & 15 & 108.13 & 8 & 20.92 & 16 & 7.59 & 7 & 0.48 & 12 & $\mathrm{C}$ \\
\hline 11 & Jiang-su & 25.53 & 6 & 3.32 & 13 & 104.24 & 13 & 25.26 & 4 & 9.47 & 17 & 0.53 & 10 & $\mathrm{C}$ \\
\hline 12 & Shanghai & 29.29 & 16 & 4.05 & 16 & 99.63 & 17 & 21.95 & 12 & 7.42 & 6 & 0.67 & 5 & B \\
\hline 13 & Fu-jian & 25.76 & 9 & 2.53 & 6.5 & 108.63 & 6 & 22.45 & 11 & 7.68 & 10 & 0.43 & 14 & $\mathrm{C}$ \\
\hline 14 & Qing-dao & 27.13 & 13 & 2.84 & 9 & 117.47 & 2 & 28.13 & 1 & 8.68 & 13 & 0.38 & 18 & $\mathrm{D}$ \\
\hline 15 & Bei-kong & 24.71 & 3 & 2.53 & 6.5 & 106.93 & 10 & 25.95 & 2 & 10.89 & 20 & 0.42 & 15 & $\mathrm{C}$ \\
\hline 16 & Tong-xi & 25.71 & 8 & 2.52 & 4 & 109.98 & 5 & 18.82 & 20 & 7.68 & 9 & 0.46 & 13 & $\mathrm{C}$ \\
\hline 17 & Ji-lin & 26.68 & 11 & 2.32 & 2.5 & 111.90 & 4 & 19.71 & 19 & 7.13 & 2.5 & 0.39 & 17 & $\mathrm{D}$ \\
\hline 18 & Tian-jin & 21.18 & 1 & 1.63 & 1 & 116.08 & 3 & 20.79 & 17 & 7.13 & 2.5 & 0.25 & 19 & $\mathrm{D}$ \\
\hline 19 & Ba-yi & 24.92 & 4 & 4.34 & 19 & 97.21 & 20 & 24.13 & 8 & 8.89 & 15 & 0.66 & 6 & B \\
\hline 20 & Fo-shan & 25.5 & 5 & 2.32 & 2.5 & 120.68 & 1 & 24.55 & 5 & 7.26 & 5 & 0.18 & 20 & E \\
\hline
\end{tabular}

Note: When the data get bigger, rank assignment gets bigger too; reversed assignment is used in indicators of lost scores and fouls. 
Table 5. Comprehensive Evaluation and Analysis of of Each Competing Team's Offensive and Defensive RSR Values in the CBA Season 2015-2016 League

\begin{tabular}{|c|c|c|c|c|c|c|c|c|c|c|c|c|}
\hline Ranking & Teams & $\begin{array}{l}\text { Offensive } \\
\text { RSR }\end{array}$ & $\begin{array}{c}\text { Offensiv } \\
\text { RSR } \\
\text { Ranking }\end{array}$ & Level & Ranking & $\begin{array}{c}\text { Defensive } \\
\text { RSR }\end{array}$ & $\begin{array}{c}\text { Defensiv } \\
\text { RSR } \\
\text { Ranking }\end{array}$ & Level & Ranking & $\begin{array}{c}\text { Offensive-defensive } \\
\text { RSR }\end{array}$ & Level & $\begin{array}{c}\text { RSR } \\
\text { Ranking }\end{array}$ \\
\hline 1 & Liao-ning & 0.74 & 4 & B & 17 & 0.73 & 2 & B & 19 & 0.9 & A & 1 \\
\hline 2 & Xin-jiang & 0.80 & 1 & A & 20 & 0.57 & 9 & B & 12 & 0.8 & A & 4 \\
\hline 3 & Si-chuan & 0.78 & 2 & B & 18.5 & 0.61 & 8 & B & 13 & 0.79 & A & 5 \\
\hline 4 & Guang-dong & 0.71 & 6 & B & 15 & 0.75 & 1 & B & 20 & 0.87 & A & 3 \\
\hline 5 & Shan-dong & 0.78 & 2 & B & 18.5 & 0.68 & 3 & B & 17.5 & 0.9 & A & 1 \\
\hline 6 & Guang-sha & 0.73 & 5 & B & 16 & 0.66 & 6 & B & 14.5 & 0.76 & B & 6 \\
\hline 7 & Beijing & 0.58 & 11 & $\mathrm{C}$ & 10 & 0.68 & 3 & B & 17.5 & 0.69 & B & 7 \\
\hline 8 & Zhe-jiang & 0.60 & 8 & B & 12.5 & 0.42 & 15 & $\mathrm{C}$ & 5.5 & 0.44 & $\mathrm{C}$ & 12 \\
\hline 9 & Shen-zhen & 0.41 & 14 & $\mathrm{C}$ & 7 & 0.53 & 10 & $\mathrm{C}$ & 10.5 & 0.45 & $\mathrm{C}$ & 11 \\
\hline 10 & Shan-xi & 0.40 & 15 & $\mathrm{C}$ & 6 & 0.48 & 12 & $\mathrm{C}$ & 9 & 0.38 & $\mathrm{D}$ & 15 \\
\hline 11 & Jiang-su & 0.47 & 12 & $\mathrm{C}$ & 9 & 0.53 & 10 & $\mathrm{C}$ & 10.5 & 0.49 & $\mathrm{C}$ & 9 \\
\hline 12 & Shanghai & 0.31 & 17 & D & 4 & 0.67 & 5 & B & 16 & 0.5 & $\mathrm{C}$ & 8 \\
\hline 13 & Fu-jian & 0.60 & 8 & B & 12.5 & 0.43 & 14 & $\mathrm{C}$ & 7 & 0.49 & $\mathrm{C}$ & 9 \\
\hline 14 & Qing-dao & 0.65 & 7 & B & 14 & 0.38 & 18 & $\mathrm{D}$ & 3 & 0.43 & $\mathrm{C}$ & 13 \\
\hline 15 & Bei-kong & 0.46 & 13 & $\mathrm{C}$ & 8 & 0.42 & 15 & $\mathrm{C}$ & 5.5 & 0.34 & D & 16 \\
\hline 16 & Tong-xi & 0.28 & 18 & D & 3 & 0.46 & 13 & $\mathrm{C}$ & 8 & 0.28 & D & 18 \\
\hline 17 & Ji-lin & 0.32 & 16 & $\mathrm{D}$ & 5 & 0.39 & 17 & $\mathrm{D}$ & 4 & 0.26 & $\mathrm{D}$ & 19 \\
\hline 18 & Tian-jin & 0.27 & 19 & $\mathrm{D}$ & 2 & 0.25 & 19 & $\mathrm{D}$ & 2 & 0.1 & $\mathrm{E}$ & 20 \\
\hline 19 & Ba-yi & 0.18 & 20 & $\mathrm{E}$ & 1 & 0.66 & 6 & B & 14.5 & 0.38 & D & 15 \\
\hline 20 & Fo-shan & 0.5 & 10 & $\mathrm{C}$ & 11 & 0.185 & 20 & $\mathrm{E}$ & 1 & 0.3 & D & 17 \\
\hline
\end{tabular}

Table 6. Ranking of of Each Competing Team in the CBA Season 2015-2016 League and RSR Rank Correlation of Offensive Indicators, Defensive Indicators, Offensive-defensive RSR Overall Ranking Correlation in the CBA Season 2015-2016 League

\begin{tabular}{|c|c|c|c|c|c|}
\hline $\begin{array}{l}\text { Match } \\
\text { Ranking }\end{array}$ & & $\begin{array}{l}\text { Match } \\
\text { Ranking }\end{array}$ & $\begin{array}{l}\text { RSR of } \\
\text { Competing } \\
\text { Teams' Offensive } \\
\text { Indicators }\end{array}$ & $\begin{array}{l}\text { RSR Rank of } \\
\text { Defensive } \\
\text { Indicators }\end{array}$ & $\begin{array}{l}\text { Offensive-defensive } \\
\text { RSR overall }\end{array}$ \\
\hline & $\begin{array}{c}\text { Person } \\
\text { Correlation }\end{array}$ & 1 & -0.807 & -0.704 & -0.902 \\
\hline & $\begin{array}{l}\text { Significance } \\
\text { (both sides) }\end{array}$ & & $0.00^{* * *}$ & 0.005 & $0.00 * *$ \\
\hline & $\mathrm{n}$ & 20 & 20 & 20 & 20 \\
\hline
\end{tabular}

Analysis of Competition Pattern of Each Competing Team in the CBA Season 2015-2016 League

In order to confirm the competition pattern of each competing team in the CBA Season 2015-2016 League, we can use probability unit "Probit" which can express the downside cumulative frequency given by RSR value to understand the distribution of each team's comprehensive offensive-defensive RSR value[6] [7]. At the same time, we can take advantage of the best classification principles and the actual situation of each competing team to classify their competition patterns. From Table 7,The specific procedures are as follows: (1) To compile RSR frequency distribution table, to list each frequency f, and to calculate accumulated frequency $\sum f ;(2)$ Todetermine each RSR's rank scope $\mathrm{R}$ and its average rank $\bar{R}$; (3) To calculate the cumulative frequency $(\bar{R} / \mathrm{n}) \times 100 \%$, and the final accumulation will be revised according to(1-1/kn); (4) Converse percentage P into the probability unit "Probit". Probit equals to the standard normal dispersion " $u$ " corresponding to the percentage P) plus 5; (5) The probability unit Probit, corresponding to cumulative frequency, is set as the independent variable and RSRI, as the dependent variable, to calculate the linear regression equation, that is, $\mathrm{RSR}=\mathrm{a}+\mathrm{b} \times$ probit. [8] 
Table 7. Each Competing Team's Offensive-defensive RSR Overall Distribution and Probit Value in the CBA Season 2015-2016 League

\begin{tabular}{cccccc}
\hline RSR & $\mathrm{f}$ & $\sum f$ & $\bar{R}$ & $\begin{array}{c}(\bar{R} / \mathrm{n}) \\
\times 100 \%\end{array}$ & probit \\
\hline 0.10 & 1 & 1 & 1 & 5 & 3.35 \\
0.26 & 1 & 2 & 2.5 & 12.5 & 3.84 \\
0.28 & 1 & 3 & 4 & 20 & 4.16 \\
0.30 & 1 & 4 & 5 & 25 & 4.33 \\
0.34 & 1 & 5 & 6 & 30 & 4.48 \\
0.38 & 2 & 6 & 7.5 & 37.5 & 4.68 \\
0.43 & 1 & 8 & 9 & 45 & 4.87 \\
0.45 & 2 & 10 & 10.5 & 52.5 & 5.06 \\
0.49 & 2 & 12 & 12.5 & 62.5 & 5.32 \\
0.5 & 1 & 13 & 13 & 65 & 5.39 \\
0.69 & 1 & 14 & 14 & 70 & 5.52 \\
0.76 & 1 & 15 & 15 & 75 & 5.67 \\
0.79 & 1 & 16 & 16 & 80 & 5.84 \\
0.8 & 1 & 17 & 17 & 85 & 6.04 \\
0.87 & 1 & 18 & 18 & 90 & 6.28 \\
0.9 & 2 & 20 & 19.5 & 97.5 & 6.94 \\
\hline
\end{tabular}

Table 8. Classification Ranking of Each Competing Team's Offensive-defensive Strength in the CBA Season 2014-2015 League

\begin{tabular}{|c|c|c|c|c|}
\hline $\begin{array}{l}\text { Classifi } \\
\text { cation }\end{array}$ & PX & probit & RSR & Result of classification ranking \\
\hline Class 3 & $<=\mathrm{P} 39.76$ & $<=4.7$ & $<=0.38$ & $\begin{array}{c}\text { Teams of Shan-xi, Bei-kong, Tong-xi, Ji-lin, } \\
\text { Tian-jin, Ba-yi, Fo-shan }\end{array}$ \\
\hline Class 2 & $\begin{array}{l}\mathrm{P} 39.76 \sim \mathrm{P} \\
\quad 67.07\end{array}$ & $4.7 \sim 5.5$ & $0.38 \sim 0.69$ & $\begin{array}{l}\text { Teams of Zhe-jiang, Shen-zhen, Jiang-su, } \\
\text { Shanghai, Fu-jian, Qing-dao }\end{array}$ \\
\hline Class 1 & $>=67.07$ & $>=5.5$ & $>=0.69$ & $\begin{array}{l}\text { Teams of Liao-ning, Xin-jiang, Si-chuan, } \\
\text { Guang-dong, Shan-dong, Guang-sha, Beijing }\end{array}$ \\
\hline
\end{tabular}

Use the Softwar $\mathrm{R}$ to obtain the relevant data. The established regression equation is $\mathrm{Y}=-0.766+0.252 \mathrm{X}$; to have a none-zero significance test of the regression equation coefficient, $\mathrm{T}=14.338, \mathrm{p}<0.01$, meaning that the calculated parameter is of statistical significance. $\mathrm{F}=205.6, \mathrm{p}<0.01$, indicates that the undertaken linear equation is of statistical significance. (6) Classification is carried out according to the obtained results combining the actual situation as well as the best classification principles.[9] From Table 8, Of the twenty competing teams in the CBA Season 2015-2016 League, teams of Liao-ning, Xin-jiang, Si-chuan, Guang-dong, Shan-dong, Guang-sha and Beijing were classified as the top seven of the League thanks to their overall Excellency in both offensive and defensive performance. So these seven teams are Class 1 teams. The six teams of Zhe-jiang, Shen-zhen, Jiang-su, Shanghai, Fu-jian, Qing-dao were Class 2 teams, while the other seven teams of Shan-xi, Bei-kong, Tong-xi, Ji-lin, Tian-jin, Ba-yi and Fo-shan were Class 3 teams.

\section{Conclusion}

RSR method was adapted to rate RSR values of all the competing teams in the CBA Season 2015-2016 League. A correlation test was done for each team's attacking, defending, as well as the final ranking of each team and its attacking and defending strength to get correlation coefficient $r$. Differences were obtained from such tests.

(1) In the evaluation of all the twenty teams' attacking strength, Xin-jiang Team was the only team whose attacking capability reached Level A. Attacking strength of 
teams of Liao-ning, Si-chuan, Guang-dong, Shan-dong, Shan-dong, Guang-sha, Zhe-jiang, Fu-jian and Qing-dao reached Level B with RSR value ranging between 0.6-0.78. There are six teams whose attacking strength was Level B. Ba-yi Team was the worst team of all the competing teams in terms of attacking strength and was classified as Level E. Relevant software was used to calculate the correlation coefficient between RSR value and league ranking, $r=-0.807(\mathrm{p}<0.01)$, which indicates a significant correlation and attacking RSR value can objectively reflect a league team's attacking capability.

(2) As for defense, there was no team in the League whose defending strength was able to reach Level A. The top seven teams, as well as Shanghai Team and Ba-yi Team's defense reached Level B, of which the best was Guang-dong Team with a defending RSR value of 0.75 and the worst was Xin-jiang Team with a defending RSR value of 0.57. These seven teams of Zhe-jiang, Shen-zhen, Shan-xi, Jiang-su, Fu-jian, Bei-kong and Tong-xi are of Level C in defense, of which the defending RSR value of Jiang-su Team, the best one in this level, was 0.53 ; and the defending RSR value of the last two teams was 0.42. The defending level of teams of Qing-dao, Ji-lin and Tian-jin was Level D, while Fo-shan's defending RSR value was as low as 0.18 and was ranked the last team of all. Relevant software was used to calculate the correlation coefficient between RSR value and league ranking, $r=-0.704(p<0.01)$, which indicates a significant correlation and means that attacking RSR value can objectively reflect a league team's defending strength.

(3) In terms of comprehensive offensive-defensive capability, the top five teams in the League reached the level of A, of which four teams were able to be the top four teams. This shows that both of their attacking and their defending strength were really comprehensive. The offensive-defensive strength of Beijing Team and Guang-sha Team was Level B. The aoffensive-defensive strength of these six teams of Zhe-jiang, Shen-zhen, Shanghai, Fu-jian, Qing-dao and Jiang-su was C, the middle level of the League; while the offensive-defensive RSR values of these five teams of Bei-kong, Tong-xi, Ji-lin, Ba-yi and Fo-shan were between 0.26 0.38, Level D. Relevant software was used to calculate the correlation coefficient between RSR value and league ranking, $\mathrm{r}=-0.902(\mathrm{p}<0.01)$, which indicates a significant correlation and that attacking RSR value can objectively reflect a league team's offensive-defensive strength.

\section{Suggestions}

(1) The overall defending strength of all competing teams in the CBA Season 2014-2015 League was as low as C, consequently, all the teams of the League must continue to keep practicing their defending capability, especially for those teams which are "strong in attacking, poor in defending". These teams must constantly pay special attention to the practice of defense in the future training and matches.

(2) In this season, to some extent, the traditionally strong teams of the League, particularly Beijing Team and Guang-dong Team, have been threatened by other teams. That was mainly caused by the aging problem existing in their core team members. These teams should learn to give younger players more opportunities in the future matches to shorten the process of replacing older players with younger ones so that the teams are able to remain competitive.

(3) For teams like Ba-yi, a representative which is "strong in defense and weak in attack", they must strengthen attacking capability from all sides. They can bring in players of stronger attacking capability; emphasize the training of basic skills of 
attacking as well as tactical cooperation. Those teams should learn to establish a style of "depending on defense combined with attack" in basketball matches.

\section{References}

[1] Ming-zhi Sun. Advanced course of modern basketball[M].Beijing:People's Sports Publishing House, 2004(In Chinese)

[2] Li-chao Zhang. Research on the Attacking Ability Differences between Major Asian Rivals with London 2012 Olympics China Men' s Basketball Team[J]. Journal of Beijing Sport University, 2011, 34 (8):132-134(In Chinese)

[3] Wei-hua Cao. A comparison of the offense and defense strengths of teams in CBA game season 2010-2011 and an analysis of the structure of competition[J]. Journal of Physical Education, 2012, 19(2):109-115(In Chinese)

[4] Mao-quan Hu, Qing-zhu Sun, Hua Rong. Comparative Study on Defensive and Offensive Ability of Each Team in the 16th World Man's Basketball Championship [J]. China Sport Science and Technology, 2011, 47(1):34-39(In Chinese)

[5] Zhen-qiu Sun. Zhenqiu Sun. Medical statistics [M]. Beijing: People's Medical Publishing House, 2005:510-520(In Chinese)

[6] Zhi-qiang Jia, et al., A Research on the Differences of the offensive and defensive capacity between China's Men's Basketball Team and Its Opponents in the 25th Asian Basketball Championship[J].Journal of Beijing Sport University,2010, 33(2):123-127 (In Chinese)

[7] Feng-tiao Tian. The problem of RSR method step[J]. Chinese Journal of Health Statistics, 1993, 10(2):25-29(In Chinese)

[8] Prineas RJ, Crow RS, Blackburn HW. The Minnesota code manual of electrocardiographic findings: standards and procedures for measurement and classification. 2nd edit. London: Springer; 2010.

[9] Bussink BE, Holst AG, Jespersen L, Deckers JW, Jensen GB, Prescott E. Right bundle branch block: prevalence, risk factors, and outcome in the general population: results from the Copenhagen City Heart Study. Eur Heart J 2013;34:138-46. 